\title{
El concepto de liderazgo en los espacios educativos: alcances y límites de un término elástico
}

\author{
The concept of leadership in educational spaces: scope and limits of an \\ elastic word
}

O conceito de liderança em espaços educacionais: alcance e limites de uma palavra elástica

\begin{abstract}
Oscar Julián Cuesta Moreno* id Emilce Moreno Mosquera** iD
* Doctor en Ciencias Sociales. Doctor en Conocimiento y Cultura en América Latina. Comunicador social. Magíster en Educación. Docente e investigador de la Facultad de Educación de la Universidad Javeriana. Director del grupo de investigación Formación, Políticas y Subjetividades. ORCID: https://orcid.org/0000-0001-7181-1183

* *Licenciada en Español y Filología Clásica y Magíster en Lingüística. Doctora en Educación. Docente e investigadora de áreas relacionadas con el campo de la didáctica de la lectura y escritura y áreas afines a la lingüística en la Facultad de Educación de la Universidad Javeriana. ORCID: https://orcid.org/0000-0003-4058-5691
\end{abstract}

\section{OPEN ACCESS $\boldsymbol{\Omega}$ \\ DOI: http://dx.doi.org/10.18634/sophiaj.} 17v.1i.1010

Información del artículo

Recibido: octubre 08 de 2020

Revisado: enero 28 de 2021

Aceptado: junio 15 de 2021

Publicado: Abril 15 de 2021

Palabras clave: liderazgo educativo, liderazgo escolar, liderazgo pedagógico, directivo escolar.

Keywords: educational leadership, school leadership, pedagogical leadership, school manager.

Palavras-chave: liderazgo educativo, liderazgo escolar, liderazgo pedagógico, director escolar.

Cómo citar: / how cite:

Cuesta, O, Moreno, E. (2021) El concepto de liderazgo en los espacios educativos: alcances y límites de un término elástico. Sophia 17(1) e1010

Sophia-Educación, volumen 17 número 1. enero/junio 2021. Versión español

\section{RESUMEN}

Se presentan los resultados de una revisión documental sobre las definiciones de liderazgo educativo presentes en los trabajos publicados sobre el tema en Iberoamérica. Se realizó una consulta en bases de datos digitales. Tras analizar 87 documentos, se estableció que ambigüedad conceptual entre el liderazgo educativo, pedagógico y escolar. Además, que el liderazgo está enmarcado administrativamente pero que no se restringe a estos espacios. Se concluye que es necesario formular teorías que constituyan al liderazgo educativo como objeto de estudio con rasgos ontológicamente definidos, para evitar distorsiones epistemológicas y acumular conocimiento sobre este tema. 
Copyright 2021 Universidad La Gran Colombia

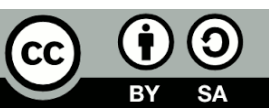

Conflicto de interés:

Los autores declaran no tener ningún conflicto de interés.

Correspondencia de autor:

oscarjuliancuesta@gmail.com moreno-e@javeriana.edu.co

\section{ABSTRACT}

The results of a documentary review on the definitions of educational leadership present in the works published on the subject in Latin America are presented. A query was made in digital databases. After analyzing 87 documents, it was established that conceptual ambiguity between educational, pedagogical and school leadership. In addition, that the leadership is framed administratively but that is not restricted to these spaces. It is concluded that it is necessary to formulate theories that constitute educational leadership as an object of study with ontologically defined features, to avoid epistemological distortions and accumulate knowledge on this subject.

\section{RESUMO}

São apresentados os resultados de uma revisão documental sobre as definições de liderança educacional presentes nos trabalhos publicados sobre o tema na América Latina. Foi feita uma consulta em bancos de dados digitais. Após análise de 87 documentos, constatou-se essa ambigüidade conceitual entre liderança educacional, pedagógica e escolar. Além disso, essa liderança é enquadrada administrativamente, mas não se restringe a esses espaços. Conclui-se que é necessário formular teorias que constituam a liderança educacional como objeto de estudo com características ontologicamente definidas, para evitar distorções epistemológicas e acumular conhecimentos sobre o tema. 


\section{Introducción}

El liderazgo se ha convertido en cualidad central para la gestión de las organizaciones. Las instituciones educativas, abocadas al cumplimiento de indicadores de calidad, han encontrado en el liderazgo un factor determinante para el cumplimiento de sus metas y el desarrollo de sus propósitos misiones de manera eficiente. El liderazgo educativo no solo se advierte como un ejercicio de gestión institucional, sino que tiene consecuencia en el aprendizaje (Bolívar, 2010; Villa, 2015). Igualmente, se ha constituido como un factor clave en la mejora de la calidad educativa (Leithwood \& Louis, 2011).

Efectivamente, el avance de las instituciones educativas depende en gran modo de los equipos directivos y el ejercicio mismo de su liderazgo en la organización, dinamización, apoyo, motivación y transformación de los procesos y estrategias institucionales. De este modo, lo evidencian el informe Mckinsey (Barber y Mourshed, $2008)$ y los informes de $\operatorname{OECD}(2008,2016)$ que incluyen el liderazgo educativo como el segundo factor relevante en el logro de aprendizaje a partir de la acción docente. Esto se debe a que los directivos que ejercen liderazgo tienen la capacidad de influir en el desarrollo y bienestar de su equipo de profesores, y por ende, en el rendimiento de los estudiantes.

Los directivos escolares son considerados en sus roles como gerentes, administradores, consejeros, es decir, se caracterizan por ejercer diferentes responsabilidadades y un liderazgo asociado a las presiones en el desempeño de sus funciones. De otro lado, hay un vínculo explícito entre la calidad y el éxito de una escuela en relación con el líder, de acuerdo con Leitwood et al. (2010):

El liderazgo se refiere enteramente a la mejora de la organización; más específicamente, se trata de establecer acuerdos y direcciones valiosas para la organización de la que se trate y hacer lo que sea necesario para empujar y apoyar a las personas a moverse en esas direcciones (p. 9).

De ahí la necesidad de pensar en los directivos docentes y el liderazgo educativo que requieren, pero ello no se puede hacer de modo aislado a la revisión bibliográfica y a las discusiones sobre el tema. Especialmente, se destaca en la actualidad el liderazgo pedagógico como esencial para generar buenas prácticas y contribuir a la mejora de resultados de aprendizaje (Leithwood, Harris, \& Hopkins, 2008; Day et al., 2011; Robinson, 2007).

Existen dos formas de influir en los resultados académicos de los estudiantes y por ende ejercer el liderazgo pedagógico (Hallinger y Heck, 1998; Leithwood, 2009). La primera se relaciona con acciones con incidencia directa en la enseñanza y el aprendizaje (la formación que aporte al desarrollo de competencias conceptuales, procedimentales y actitudinales de los directivos). La segunda forma tiene que ver con las actividades que influencian de modo indirecto las prácticas docentes (condiciones organizativas de la escuela). En ese sentido, estudios empíricos que teoricen sobre cómo las prácticas de liderazgo son moldeadas y fortalecidas por este tipo de prácticas aportan al conocimiento pedagógico por parte del equipo directivo con foco en el desarrollo profesional. Así el liderazgo se comienza a ver como una práctica en comunidad, distribuida, más democrática, estructurante en la escuela y no exclusiva de algunos.

De hecho, no solo es el liderazgo sino el estilo de liderazgo el que influye sobre la calidad y el éxito de los programas (Pedraja-Rejas, Rodríguez-Ponce y Villarroel, 2012; Robinson, Lloyd y Rowe, 2014). Lo que ha llevado a pensar diferentes perspectivas del liderazgo en las organizaciones educativas, entre otras se pueden nombrar, el liderazgo dialógico (Redondo-Sama, 2015), el socioformativo (Hernández, Tobón y Vázquez, 2015), el transformacional (Salazar, 2006), distributivo (Carreño, 2008), el formativo (Rodríguez y Mármol, 2009), el liderazgo educativo o pedagógico (Bolívar, 2010; Hallinger, 2005; Leithwood, 2009) escolar (Weinstein, 2009) y directivo (Anderson, 2010; Weinstein, 2009).

Sin embargo, a pesar de que el liderazgo educativo ha sido abordado en tesis de doctorado y maestría e, incluso, ha sido abordado por documentos de organizaciones que tienen incidencia en la educación mundial como la Organización para la Cooperación y el Desarrollo Económicos (OCDE, 2009; Pont, Nusche y Moorman, 2008), es un concepto problemático, toda vez que no es sencillo de conceptualizar (Vázquez, Bernal y Liesa, 2014).

En efecto, la definición de liderazgo educativo es difusa, dado que no existe una conceptualización homogénea. Aún más, algunos investigadores hablan de liderazgo escolar y otros de liderazgo pedagógico como sinónimos del liderazgo educativo, pero otros señalan diferencias entre ellos. Lo que, consecuentemente, aumenta la ambigüedad y amplía la imprecisión del concepto. 
Precisamente, el artículo presenta los resultados de una revisión documental para establecer qué definiciones de liderazgo educativo se expresan en los trabajos que han abordado el tema. Además, la investigación buscó ubicar en estos documentos elementos recurrentes que se estipulan debe tener un líder educativo con el fin de aportar a un marco para la buena dirección.

\section{Metodología}

Se realizó una revisión documental de carácter descriptivo, determinada por un corte temporal y geográfico. El criterio temporal estuvo determinado por trabajos publicados en los últimos 20 años. Frente al criterio geográfico se limitó a trabajos Iberoamericanos. La búsqueda se desarrolló en tres bases de datos (DIALNET, REDALYC, PROQUEST). En la búsqueda se aplicaron criterios de restrictivos en el idioma (solo español) y en el tipo de documento (artículos). Sin embargo, al final se incluyeron algunas tesis doctorales y libros que las bases de datos igual seleccionaron en la búsqueda. Igualmente, se consultaron textos en inglés que son referente para este tema a nivel internacional. Se seleccionaron para el análisis 87 documentos, cuya información se organizó en una matriz que clasificaba los trabajos según su temática.

\section{Resultados}

La revisión bibliográfica evidencia que el abordaje del liderazgo educativo se constituye en una prioridad en las agendas educativas internacionales. Este liderazgo está ligado espeialmente al rol del director y el equipo directivo y su influencia en el desarrollo y mejoramiento de la escuela. En ese orden, los documento señalan que estos deben ser realmente agentes de cambio. Otro tipo de liderazgo mencionado de manera significativa es el rol del profesor, sobre todo en su papel de gestión en la consecusion de los aprendizajes de los estudiantes.

En los documentos el liderazgo tiende a entenderse de modo holístico y ligado a los contextos educativos organizacionales, es decir, que es resultado de la concatenación de varias actitudes, comportamientos, decisiones, pero tambien sinergias interpersonales. En esa línea, los resultados de la revisión permiten advertir que es necesario disgregar la imagen habitual del liderazgo, esto es, que no se entienda como exclusivo y atribuible a la mística de una persona o la naturaleza de un cargo.

En efecto, más allá que el liderazgo sea un atributo visible en actitudes o conductas individuales, los documentos revisados lo describen y analizan como una dinámica consustancial al contexto, en este caso, a la escuela, lo que implica no disgregar los líderez y sus cualiades de las problemáticas, las transformaciones y las necesidades que interpelan la función y el rol del directivo y su equipo.

Varios documentos siguen las disposiciones internacionales de para el liderazgo educativo, de allí que se hable de que liderazgo está ligado al mejoramiento escolar. Sin embargo, tal mejoramiento es basicamente la optimización de los procesos de aprendizaje (learning-centered leadership). Por ello le atribuyen mucha importancia de la evaluación de los resultados de aprendizaje, los desempeños de los estudiantes y su relación con la efectividad del liderazgo educativo (Robinson, 2011). Consecuentemente, una crítica a esa noción de aprendizaje pasaría por dudar de la centralidad del aprendizaje, toda vez que en el acto educativo ocurre una formación del sujeto que no es reductible a un listado de aprendizajes esperados.

Por otro lado, la revisión de los documentos permitió establecer que existe una dispersión en el propio concepto de liderazgo, es decir, no hay definición compartida que permita establecer escencia sobre el mismo. De allí que varias veces se hable indistintamente de liderazgo educativo, pedagógico y escolar, pero otra veces se planteen como asuntos distintos.

En general, el liderazgo se asume como un atributo del rol del directivo escolar, por lo que varios documentos señalan los rasgos que debe tener la dirección educativa y los diferentes tipos de liderazgos vinculado a estos cargos en la pirámide organizacional de las instituciones educativas.

En el siguiente apartado se categorizan estos hallazgos en este orden: en primer lugar se analiza el concepto de liderazgo y se discute las posibles deferencias y consecuencias de su adjetivación, es decir, que implica hablar de liderazgo escolar, educativo o pedagógico. En segundo lugar, se muestran cómo los documentos abordan el liderazgo como un asunto de los cargos directivos. Con estos datos se plantea, finalmente, unas claves para un marco de buena dirección. 


\section{Discusión}

\section{Concepto de liderazgo educativo}

Varios de los documentos revisados abordan el concepto de liderazgo para ubicar sus investigaciones teóricamente. De allí que sea pertinente recuperar tales definiciones para delimitar elementos clave del liderazgo y, a partir de ello, observar lo que sería el liderazgo educativo.

En el caso de Bernasconi y Rodríguez-Ponce (2018) sostienen que el líder es quien tiene influencia en los miembros de un equipo u organización para que efectúen las tareas necesarias para conseguir los objetivos. En consecuencia, el liderazgo sería "la capacidad de influir, incidir o inducir a los grupos o individuos al interior de un equipo o una organización para lograr una determinada actuación o comportamiento en favor de alcanzar los objetivos o metas institucionales" (p.31). Castro, Miquilena y Peley (2006) coinciden en señalar que el liderazgo implica influir en los otros, pero señalan que es un fenómeno resultado de la interacción social y está presente en todos los grupos humanos.

En esa línea, Reviriego (2013) sostiene que el liderazgo es "la capacidad que posee una persona de influir sobre los miembros de una organización induciéndolos a comportarse de una determinada manera”, y en el marco de una organización "el liderazgo debe estar orientado a conseguir los fines que esa institución se propone" (p.439)

Según Navarro-Corona (2016) se pueden identificar tres enfoques en la comprensión del liderazgo: 1- uno que se centran en los rasgos personales, que parten del supuesto de que los líderes poseen características innatas, por lo que buscan analizar atributos del sujeto; 2- uno que aborda el liderazgo como el "conjunto de acciones y estrategias emprendidas por el líder para obtener éxito" (p.57), lo que lleva a pensar prácticas eficaces de los líderes; 3- uno que centra su interés en los vínculos y los relaciones entre líderes y seguidores, lo que implica incorporar las características contextuales de la institución en la que se genera el liderazgo.

Esto último, ratifica que el liderazgo debe comprenderse como proceso de orden social, es decir, "de relaciones entre los sujetos que se estructuran no solo a partir de las cualidades extraordinarias de su líder sino también de su trabajo, y de que estos dos elementos concuerden con las características y las necesidades de los seguidores y seguidoras, y con las características del contexto" (Navarro-Corona, 2016, p.62). Es decir, que el liderazgo se ve como un proceso a través del cual los individuos ejercen una influencia sobre otros, inspiran, motivan y orientan para que cumplan los objetivos como equipo (Davis y Newstrom, 2003; Leithwood et al. 2004).

Ahora bien, en el marco educativo, el liderazgo se socia a la gestión de las instituciones educativas para lograr su eficiencia, sobre todo en que los estudiantes logren aprendizajes (Badillo y Arroyo, (2015) y, en esa vía, alcanzar dinámicas de calidad (Cantón y Arias, 2008; Alonso, 2012). Esto exige no solo una capacidad de influir en el equipo de trabajo, sino también de dirigir y poseer "una visión de lo que es y debería ser la organización educativa" (Vázquez, Bernal y Liesa, 2014, p. 94).

En todo caso, si el liderazgo es la influencia que logra que un grupo de personas colaboren voluntariamente y con entusiasmo en la consecución de algún objetivo, en el liderazgo educativo puede entenderse que tal influencia está orientada en el logro del proceso de enseñanza aprendizaje (Vázquez y Rodríguez, 2016).

\section{Diferencias entre liderazgo educativo, pedagógico y escolar}

En algunos artículos se suele usar los conceptos de liderazgo escolar y liderazgo pedagógico como sinónimos de liderazgo educativo. Sin embargo, existen otros que señalan diferencias, aunque estas no siempre queden explícitas en la enunciación. Por ejemplo, Hernández, Catrinao y Bravo (2017) afirman que "el líder escolar debería comprender los principios pedagógicos que subyacen al proceso de aula" (p.542). Es decir, queda latente la idea que no siempre el líder escolar conoce de pedagogía, por lo que no es automáticamente un líder pedagógico.

Otro ejemplo es el programa Improving School Leadership de la OCDE, el cual muestra que el desarrollo del liderazgo escolar pasa por cuatro ejes principales: “(re)definir las responsabilidades; distribuir el liderazgo escolar; adquirir las competencias necesarias para ejercer un liderazgo eficaz; y hacer del liderazgo una profesión atractiva" (Bolívar, 2010, p. 15), todo ello teniendo presente que "el liderazgo para el aprendizaje es el carácter fundamental del liderazgo escolar" (Pont et al., 2008, p. 10). Como se puede advertir, nada de lo expuesto explicita lo pedagógico como dimensión constitutiva del liderazgo escolar (hablar de aprendizaje no es necesari- 
amente hablar de pedagogía pues remite a un sustantivo que se puede alcanzar sin la intervención del profesor y, de hecho, sin necesidad de la escuela), por lo que el líder escolar no es por constitución un líder pedagógico.

En esa medida, el liderazgo pedagógico implica también una mejora en la calidad y efectividad del funcionamiento de la escuela (Bolívar, 2010; Martínez-García \& Gil-Flores, 2018). Este tipo de liderazgo requiere que las instituciones focalicen sus actividades en aquello que les permita establecer sus propósitos en el equipo de profesores y directivos, de manera que se generen condiciones operativas y organizacionales para ejercer una cultura de colaboración. Igualmente, se promueve el desarrollo profesional docente (Hallinger, 2005), por ende, el liderazgo pedagógico es un liderazgo para el aprendizaje (Ord et al., 2013).

El liderazgo educativo se asume como un proyecto compartido en el cual están implicados los lideres, los colaboradores, el contexto, el proyecto y los compromisos comunes. A la manera como lo plantea Lorenzo (2005) "la función de dinamización de un grupo o de una organización para generar su propio crecimiento en función de una misión o proyecto compartido" (p. 371). Leithwood (2009) plantea que este liderazgo se asume como “(...) la labor de movilizar e influenciar a otros para articular y lograr las intenciones y metas compartidas de la escuela" (p. 20). Este liderazgo, entonces, no es atribuible a un líder sino a las acciones y formas de trabajo colaborativo que genera de modo contextualizado e inscrito más allá de la escuela, es decir en cualquier institución social. En contraste con el liderazgo escolar que sí se instalaría en esta.

El liderazgo escolar se asocia a las dinámicas de la escuela y las formas de influir en la solución de problemas (Bolívar et al., 2013), se trata de un concepto polisemántico ligado a las diferentes funciones del directivo. Es decir, asociado al trabajo ejercido por el equipo directivo en colaboración con los maestros el cual se vincula a la gestión de recursos humanos, a la gestión del clima organizacional y de convivencia, a la administración y al liderazgo para el aprendizaje (Bolívar, 2013; Pont et. al. 2008).

Diferentes investigaciones (Hallinger, 2014; Hattie, 2015; Leithwood et al. 2009) evidencian que la dirección escolar ejerce resultados considerables en el funcionamiento, los resultados y el desarrollo de la escuela. También se trata de impactar los resultados a través de intervenciones centradas en los profesores y sus capacidades (Louis et al., 2011; Pont et al., 2008; Robinson et al., 2009). Se evidencia, entonces, que el liderazgo no es un ámbito de actuación y de competencia exclusivo de los directivos, sino que realmente ha de ser ejercido por todos los miembros de la comunidad educativa.

En el estudio de la OCDE en torno al liderazgo escolar (Pont et. al., 2008) presentó evidencia sobre prácticas de 22 sistemas educativos y se encontraron responsabilidades específicas de los directores que aportan a la mejora de los resultados de estudiantes y de la escuela, estas son:

Figura. Responsabilidades de los directivos docentes desde la visión del liderazgo escolar

\begin{tabular}{|c|c|}
\hline $\begin{array}{l}\text { Apoyo calidad } \\
\text { docente }\end{array}$ & $\begin{array}{l}\text { - Se reconoce la calidad docente como factor determinante de los resultados de los } \\
\text { estudiantes, además de las responsabilidades asociadas a coordinar el curriculo y el } \\
\text { programa de enseñanza, evaluar y acompañar las prácticas docentes, fomentar la formación } \\
\text { profesional docente en la escuela y sobre todo desarrollar culturas de trabajo colaborativas } \\
\text { dentro y fuera de las escuelas. }\end{array}$ \\
\hline $\begin{array}{l}\text { Objetivos y } \\
\text { evaluación }\end{array}$ & $\begin{array}{l}\text { - Implica alinear los objetivos de aprendizaje con los estándares y } \\
\text { el currículo. Se usan datos de los desempeños para medir el } \\
\text { progreso, las habilidades de los estudiantes y desarrollar } \\
\text { sistemas inteligentes de evaluación. }\end{array}$ \\
\hline
\end{tabular}




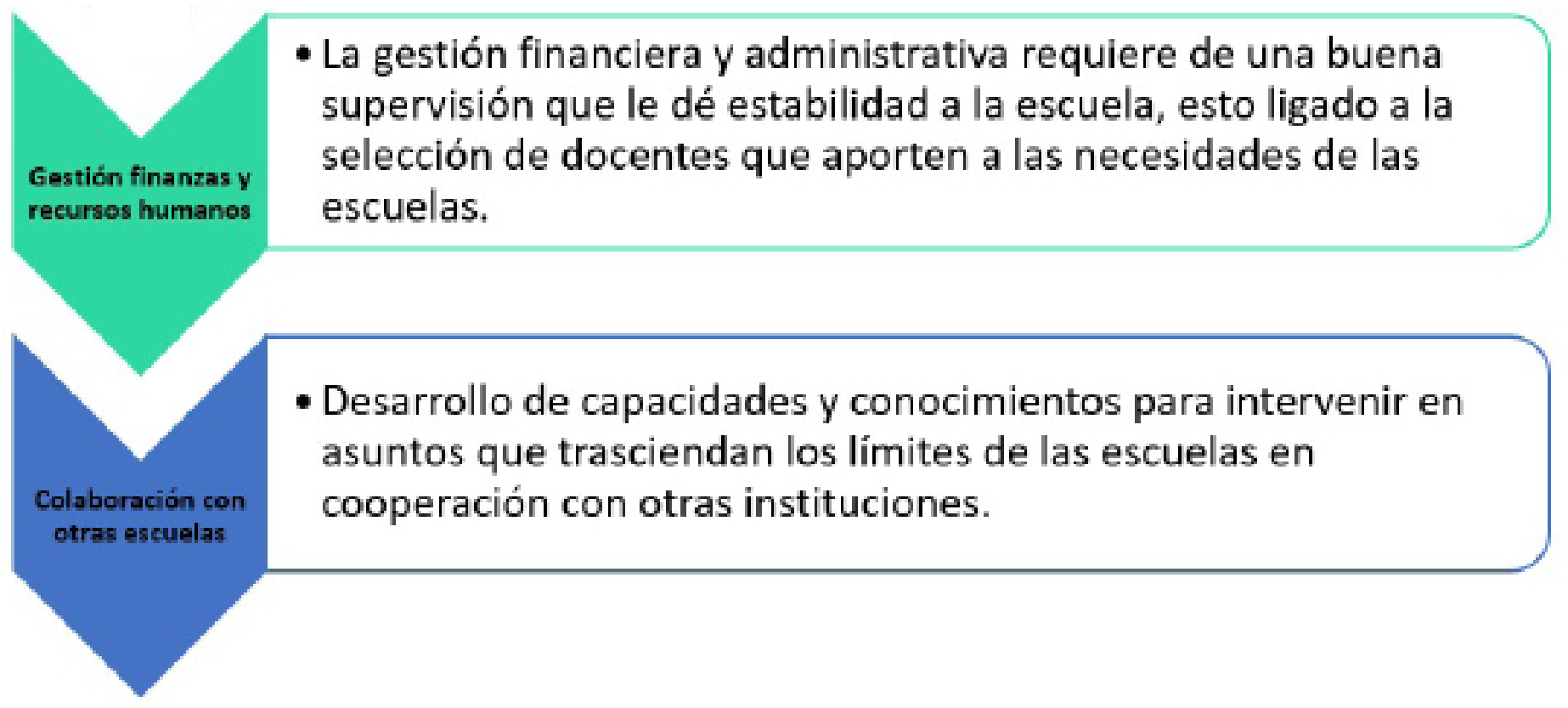

Fuente. OCDE (Pont et. al. 2008)

A partir de la revisión se puede afirmar que el liderazgo educativo es un concepto que remite a la influencia que se ejerce para en grupo para lograr con éxito el proceso educativo, pero dado que la educación no se limita a una sola institución social (universidades, medios de comunicación, familia, etc.), el liderazgo escolar indicaría que tal influencia se hace específicamente en los colegios y escuelas. Por su parte el liderazgo pedagógico haría referencia a la especificidad de influir en el currículo y la práctica de enseñanza usando el conocimiento de la pedagogía como campo del saber.

El liderazgo escolar abarca la gestión escolar, lo que implica administración y gerenciamiento de recursos ajenos al aula (aunque influyen contingentemente en la práctica que allí ocurre, pues si no hay presupuesto para el tablero esto influye en el desarrollo de la enseñanza). Si bien el informe TALIS (OCDE, 2009) muestra que no hay tensión entre un ejercicio administrativo y pedagógico, no necesariamente todo líder administrativo es un líder pedagógico y viceversa.

Esto último, implica pensar que el liderazgo escolar podría remitir al liderazgo pedagógico, pero no es consustancial a este, ya que un líder escolar podría ser un buen gerente, mas no necesariamente un pedagogo y, del mismo modo, habría buenos líderes pedagógico pero que no tienen injerencia en decisiones administrativas de las escuelas donde trabajan. Es decir, es posible pensar una escuela con líderes pedagógicos y líderes escolares que se complementan, para no caer en mesianismos que colocan una gran cantidad de cualidades en un solo actor escolar.

En síntesis, el liderazgo educativo remite al liderazgo efectuado en dichas instituciones, por lo que puede ser ejercido de diferentes formas, una de ellas sería el liderazgo pedagógico (Fernández, Palacios y Gutiérrez, 2016), el cual estaría centrado en asuntos curriculares y de los resultados de aprendizaje, lo que remite a la de planificación, organización y evaluación (Rodríguez-Molina, 2011). En esa medida, en la educación se podrían dar liderazgos administrativos (gestión de la organización), liderazgos más centrados en la naturaleza del acto de educar (pedagógicos) o el liderazgo escolar que denota una evolución del liderazgo más centrado en las necesidades, las problemáticas y los resultados de las escuelas, vistos no solo desde la función del directivo, sino desde la articulación de este con su equipo de docentes.

\section{Liderazgo como rol del director}

Varios documentos van a colocar el liderazgo como un ejercicio que recae en el director educativo o escolar. Es decir, como si fuera una actividad a ejecutar por la naturaleza del cargo. De hecho, algunas políticas educativas tienen como estrategia fortalecer el liderazgo de los directivos escolares (Monarca, 2013). Tanto así que existen trabajos que plantean el liderazgo directivo como una de las rutas más efectivas para el contexto escolar, ya que el director tiene, al final de cuentas, un poder de decisión (Fundación Empresarios por la Educación, 2018). 
Además, existen investigaciones que demuestran que el papel del liderazgo directivo es fundamental para lograr estándares de calidad, resultados eficaces (Thieme y Treviño, 2012) y cambios que mejoran la calidad de Ios aprendizajes (Ahumada, 2012). De allí que Risco (2015, p.97) afirme que: “Una de las características más importantes de las escuelas eficaces, también consideradas de calidad, es el liderazgo claro y abierto del director o directora".

En esa línea, Gento (2013) sostiene que el liderazgo del director debe ser eminentemente pedagógico, por lo que su preocupación fundamental "ha de ser la promoción o empoderamiento de todos los miembros e implicados en la entidad o iniciativa educativa, para lograr la efectividad y mejora de la calidad de la educación" (p.42).

En todo caso, es indiscutible que, en una estructura piramidal, el directivo cumple una función sustantiva y las cualidades del liderazgo son deseables para "gestionar los conflictos, acoger la diversidad de voces y permitir que estas sean escuchadas desde la jerarquía superior" (Rodríguez, 2016, p. 232). De hecho, muchas veces la cultura vertical arraigada en las instituciones educativas hace que los sujetos deseen un liderazgo directivo decisorio, contrario a teorías que hablan de participación y horizontalidad (Franco, Ortega y Ortiz, 2016), de liderazgo compartido, donde cualquier trabajador pueda ser líder y coadyuvar a la dinámica de la escuela (Salazar, 2006) o de liderazgo distribuido, basado en la interacción más que en un poder unipersonal (Maureira, Garay y López, 2016).

Ahora bien, sea el liderazgo un asunto asociado a un cargo (el director escolar) o no, lo cierto es que se advierte que es un ejercicio necesario en la educación contemporánea, toda vez que esta es una dinámica efectuada entre grupos de personas, por lo que "se reclama alguien que sepa responder a todas las cuestiones que justifican el sentido de este grupo, en nuestro caso, el aprendizaje y desarrollo personal y social de cada individuo y, por ende, de esa misma comunidad" (Corbella, 2014, p.96).

De otro lado, en la literatura se encuentran estudios que señalan que el liderazgo es necesario para que las instituciones se adecuen a las necesidades sociales (Alatorre, 2013), lo que implica tener un referente ético en la sostenibilidad (Sierra, 2016), dominar habilidades y actitudes que responden a los nuevos cambios (Castro, Miquilena y Peley, 2006) y comprometerse con la inclusión (Fernández y Hernández, 2013). En esa línea, el liderazgo es una cualidad de la organización que es sistémica y distribuida (Bolívar, 2000; Leithwood et al.2009). Es decir, un liderazgo múltiple, en equipo, compartido, en el que los docentes asuman su papel como profesionales en sus áreas y ejerzan la dirección de sus campos de conocimiento, para ello, el cuerpo profesoral tendrá que comprometerse a:

- $\quad$ El desarrollo de una visión compartida centrada en los estudiantes y sus necesidades.

- Un compromiso e implicación en la enseñanza y el aprendizaje.

- $\quad$ Una implicación del profesorado por el aprendizaje profesional de todo el personal.

- El desarrollo de una cultura de colaboración.

- Un énfasis en el aprendizaje en acción.

- Facilitar sistemas de pensamiento. (Sheppard et. al. 2009, p. 16).

El directivo se convierte así en un agente de cambio que inspira y capitaliza las competencias de los miembros de su comunidad para lograr fines comunes, resolver los problemas de la escuela, establecer metas y desarrollar un clima de colaboración.

\section{Dirección educativa y tipos de liderazgo}

Frente al papel que cumplen los directivos y el liderazgo que deben ejercer estos se han desarrollo reflexiones y se han traído teorías de otros campos para determinar cualidades que es necesario considerar en el cargo. Por ejemplo, García y Flores (2018) señalan la importancia del liderazgo distributivo y muestran que: "se caracteriza por que el líder crea rutinas que ayudan a transformar la cultura del centro, contribuyendo a la mejora de la satisfacción laboral de los docentes y del desempeño académico en el alumnado, con una pérdida de protagonismo para las funciones y acciones del líder" (p.80). Por lo que se asocia a formas democráticas y de corresponsabilidad (Ojeda, 2007). Por ejemplo, Pérez, Romero, Calderón y Verdú (2012) muestran la importancia del liderazgo situacional del profesor para el aprendizaje de los estudiantes. 
Otro de los tipos de liderazgo que es abordado en los documentos es el transformacional, "dicho liderazgo se enfoca en motivar a las personas a imprimir su mayor esfuerzo en el logro de sus expectativas. Se producen cambios en los grupos, las organizaciones y la sociedad, los cuales representan a su vez, un beneficio para la colectividad" (Bracho y García, 2013, p.167), para lo cual el líder "genera propósitos y metas que son desafiantes, las que se procuran alcanzar como institución o como equipo" (Bernasconi y Rodríguez-Ponce, 2018, p.31). En ese orden, se trata de ver el liderazgo no como un asunto de una persona sino de una organización, lo que Monarca (2013) llama liderazgo organizacional.

Por su parte, Navarro-Granados (2017) plantea el liderazgo para la justicia social que "trata de aquel liderazgo que centra sus acciones, en primer lugar, en el reconocimiento de la desigualdad de aquellos grupos con riesgo de exclusión social" (p.165), y que busca incidir en los procesos de enseñanza y aprendizaje de manera eficaz. Este liderazgo implica un reconocimiento de las desigualdades y tener presente la naturaleza de las escuelas en contextos desfavorecidos (Llorent-Bedmar, Cobano-Delgado y Navarro-Granados, 2017). Se trata de un liderazgo orientado a las prácticas de los equipos directivos para lograr una cultura inclusiva que elimine la marginalización de raza, clase, género, condiciones de aprendizajes especiales, que trabaje por el bien común y una justicia distributiva (Murillo \& Hernández, 2014; Theoharis, 2007; Tintoré, 2018).

El liderazgo para la justicia social comparte ciertos rasgos con el liderazgo 'formativo' propuesto por Rodríguez y Márnol (2009), ya que habla de "amor por el conocimiento, el compromiso, el respeto, el cuidado y la responsabilidad” (p. 202). Incluso, se relaciona con el liderazgo emocional (Goleman, 2014) en el que el líder puede influir en las emociones del equipo para formentar el conocimiento de sí, la autorregulación y las habilidades sociales.

\section{Claves para un marco de buena dirección}

Ahora bien, para algunos trabajos ser líder o ejercer el liderazgo no trae consustancialmente cualidades positivas, dado que algunos de ellos adjetivan la labor. Es decir, existen características que orientan el liderazgo para lograr una buena dirección. Por ejemplo, Alonso (2012) habla de liderar con eficiencia, para lo cual hay que ser agente de cambio permanente y coordinar las acciones entre los actores (Alonso, 2012). Por su parte, Vivolo (2015) plantea la idea de líder creativo, que no se subsume a lo imperante y se adapta al entorno.

En ese orden, hay que señalar en primer lugar que el liderazgo del directivo no se limita a su gestión administrativa, puesto que su función puede alentar o dificultar el trabajo de los docentes y, por tanto, el del aprendizaje de los estudiantes (Ramos, 2015). De allí que otro aspecto que los directivos deben dominar para ejercer un liderazgo adecuado es mantener un buen clima escolar, lo que pasa por tener inteligencia emocional y mantener buenas relaciones interpersonales a partir de la comunicación (Jiménez, 2017). Sin embargo, algunas de estas cualidades para el liderazgo directivo pasan por una dimensión carismática (Thieme y Treviño, 2012), o por las relaciones que mantienen las personas en el seno de las comunidades o instituciones a las que pertenecen (Sánchez y Hernández-Castilla, 2014).

Incluso, hay trabajos que señalan que las cualidades para el liderazgo estarían cruzadas por la condición de género y que las mujeres tendrían características para efectuarlo de mejor manera:

Si consideramos que las mujeres tienden a un tipo de liderazgo más consensuador, más de colaboración, en el que se prioriza crear un clima de entendimiento y participación, donde se fomenta la comunicación, el debate, el diálogo y el trabajo en equipo, cómo es posible que haya tan pocas mujeres en puestos directivos por elección de sus compañeros y compañeras (Díez, Valle, Terrón y Centeno, 2003, p.15).

Además, algunos autores abogan por cambiar verticalidad de la escuela, modificando la forma jerárquica y logrando relaciones entre líderes y profesores de tipo ascendentes, descendentes y lateral, haciendo énfasis en la cultura colaborativa (Cantón y Arias, 2008). Entre otras cosas, la fragmentación de la dinámica vertical pasa por la misma definición de liderazgo como influencia, pues implica aceptar que "puede ser ejercido tanto por actores con cargos formales en la organización, como por personas que no los tienen, pero que logran influir en algún curso de acción que involucra a otros, en línea con el norte común" (Horn y Marfán, 2010, p. 84).

Ahora bien, si bien existe la idea de un liderazgo compartido o que pueden existir varios actores con potencial de liderazgo, lo cierto es que la forma y disposición de las instituciones escolares tiende a colocar al directivo (rector, coordinador, etc.) en posición de líder. De allí que es importante que el director pueda ser capaz de atraer al equipo "docente, administrativo, de mantenimiento; además de los padres, representantes y comunidad para llevar a cabo las acciones propuestas en consenso, en función de viabilizar el proceso educativo en lo ped- 
agógico, la gestión y lo comunitario, para alcanzar la calidad” (Rangel y Vitorá, 2016, p.209).

Por otro lado, en algunos documentos se advierte que el liderazgo es una responsabilidad del individuo y que sus acciones son determinantes para comprometer a los colaboradores en la consecución y desarrollo de proyectos (Sánchez, 2014), por lo que el director debe, además de administrar los recursos, promover "en el colectivo escolar, el interés, la participación y el compromiso para la construcción del sentido de esa escuela, con el fin de mejorar los aprendizajes de sus estudiantes" (Rodas y Sipión, 2014, p.13).

Esto último permite mostrar la importancia de que el liderazgo no solo se efectúe en la escuela como estructura singular, sino que se haga en red, como es la idea de liderazgo de sistema, donde se integran varios líderes de diversas instituciones y trabajan comunitariamente (Delgado, 2012). En todo caso, no se trata solo de influir, sino hacerlo para que la gente se empeñe en lograr objetivos voluntariamente, lo que puede llamarse el principio de la voluntariedad (Vallejo, Chancay y Mosquera, 2016).

En una sociedad cambiante y plural como la nuestra los roles y las funciones del directivo docente se transforman y actualizan constantemente para dar alcance a las diferentes demandas y problemáticas, por ello, el conocimiento sobre el liderazgo que puedan ejercer los directores y equipos docentes se constituye en una fuente importante para el establecimiento de un marco o unos acuerdos desde la política de cara a comprender el rol del directivo ligado a los desafíos de los procesos sociales, culturales y educativos actuales. A continuación, se sintetizan algunos puntos implicados en dicho rol:

1. Un líder educativo debe, sobre todo, suscitar la intervención activa de todos los miembros de la institución, lo cual significa que su figura es doble en cuanto se mantiene como eje articulador de las dinámicas de la organización y, sin embargo, su posición debe ubicarse dentro de la horizontalidad de la base conformada por toda la comunidad de su institución.

2. La función de un líder pedagógico es encaminar y promover una auténtica educación de calidad dentro de una perspectiva de mejora continua de la propia institución y en los procesos que llevan a cabo de cara a buscar mejores resultados a nivel de la gestión, la administración de recursos y del capital humano. Para este caso, el líder directivo debe considerar:

- La comunicación

- La motivación

- La apertura al cambio

Lo anterior, en términos de éxito de la organización, permite al líder directivo formar equipos, facilitar el consenso, promover la lealtad y orientar al personal para desarrollar las actividades de la mejor manera (Furguerle Rangel \& Vitorá, 2016, p. 210).

3. Una relación, según los dos puntos anteriores, entre un centro (particular o colectivo) y un contexto, según Gento (2013):

- Establece un nexo de la política educativa con la práctica escolar y relaciona el centro con su entorno.

- Influye en la mejora del clima interno o ambiente escolar.

- $\quad$ Promueve la utilización estratégica de los recursos disponibles.

- Mejora la motivación de los profesores y contribuye al éxito de los estudiantes.

- Impulsa innovaciones educativas de éxito y promueve organizaciones de aprendizaje. (p. 45).

Tomando como marco estos ámbitos de actuación, el líder educativo coordina sus esfuerzos para alcanzar las metas de su institución, pero lo hace en equipo. Como gestor también debe asegurar el objetivo principal de la escuela y vincular, así, liderazgo, aprendizaje y calidad educativa. También los procesos directivos se orientan a distribuir y administrar los recursos humanos, financieros y materiales para conseguir las metas de aprendizaje, lo cual implica, entre otros el fortalecimiento del desarrollo profesional de los docentes, la organización del trabajo en equipo y la creación de unas condiciones óptimas de cooperación. Esto sin contar con el conocimiento de la normativa legal que le permite tener un marco de comprensión de la política educativa que lo rige y le 
permite viabilizar las acciones, los procesos y los procedimientos en la escuela. Adicional a esto se encuentra el aporte al clima organizacional, de convivencia y las relaciones interinstitucionales, fundamentales para fortalecer la comunicación interna y establecer redes de apoyo.

\section{Conclusiones}

En primer lugar, siguiendo a Vázquez y Rodríguez (2016), se puede concluir que "existen diversos criterios acerca del liderazgo educativo; de ahí que es difícil encontrar unanimidad en su conceptualización” (p. 99). Esto se debe a que "está atravesado por procesos sociales, económicos, políticos y culturales que determinan su identidad" (Fundación Empresarios por la Educación, 2018, p.32).

No obstante, al ser término polisémico que admite múltiples significados (Vázquez, Bernal y Liesa, 2014), se presentan dificultades ontológicas y epistemológicas que hacen dudar de la forma en que se construyen las investigaciones sobre el liderazgo educativo. En efecto, si no hay un cierre en la definición que sea aceptado por la comunidad de investigadores difícilmente se logrará compilar saber riguroso sobre la materia. En ese orden, es pertinente formular teorías que procuren constituir al liderazgo como objeto de estudio con rasgos ontológicamente definidos, de lo contrario seguirá siendo un concepto elástico que, incluso, para la fecha que se efectuó está investigación, no está considerado en el Tesauro de la UNESCO.

Por otro lado, se cuestiona la idea de liderazgo educativo centrada en la lógica corporativa o empresarial, pues lleva a pensarlo desde esquemas de interpretación que encasilla a los sujetos y las instituciones (Monarca, 2013). A este respecto, Pareja Fernández de la Reguera (2009) argumenta que es riesgoso llevar comprensiones del liderazgo administrativo a la escuela porque esta es una estructura compleja y porque es importante la autonomía el profesor. En esa línea, Fernández, Palacios y Gutiérrez (2016) sostienen que el liderazgo en una "institución educativa debe ser eminentemente pedagógico" (p.134) por lo que sus características deben adecuarse a la naturaleza del acto educativo. En ese orden, cabe preguntarse: ¿Qué debe incluirse en la política para lograr un liderazgo educativo que aporte de modo situado a la escuela? y ¿qué se requiere en términos de capacitación y formación de directores y líderes educativos?

Si bien el liderazgo puede estar orientado a la gestión de las organizaciones educativas, lo que implica varias tareas, lo cierto es que su meta u objetivo natural es mejorar los aprendizajes de los estudiantes (Hallinger y Heck, 2014). Si bien algunos hablan de alcanzar la calidad, la excelencia o el éxito educativo, todos estos propósitos remiten indefectiblemente al estudiante. Sin embargo, son pocos los trabajos que tienen en cuenta en el análisis del liderazgo a los estudiantes como informantes, por lo que una posibilidad es efectuar encuestas, entrevistas, grupos focales, donde ellos den cuenta de lo que hace un buen líder.

\section{Referencias bibliográficas}

Ahumada, L. (2012). Liderazgo en organizaciones educativas. Persona (15), 239-252.

Alatorre, A. V. (2013). Interdependencia entre el liderazgo transformacional, cultura organizacional y cambio educativo: una reflexión. REICE. Revista Iberoamericana sobre Calidad, Eficacia y Cambio en Educación, 11(1), 73-91.

Alonso, M. (2012). Liderar en escuelas normales que aprenden. Responsabilidad y calidad. RIDE Revista Iberoamericana para la Investigación y el Desarrollo Educativo, 3(5), 111-122.

Badillo, J. M. R., \& Arroyo, J. C. (2015). La importancia de la gestión y el liderazgo escolar en las instituciones de Educación Media Superior Tecnológica (CETIS y CBTIS) del estado de Tlaxcala. RIDE Revista Iberoamericana para la Investigación y el Desarrollo Educativo, 6(11), 359-380.

Barber, M. y Mourshed, M. (2008). Cómo hicieron los sistemas educativos con mejor desempeño del mundo para alcanzar sus objetivos. Mckinsey \& Company. PREAL. Disponible en: http://www.oei.es/pdfs/documento preal41.pdf

Bernasconi, A., \& Rodríguez-Ponce, E. (2018). Análisis Exploratorio de las Percepciones sobre los Estilos de Liderazgo, el Clima Académico y la Calidad de la Formación de Pregrado. Formación universitaria, 11(3), 29-40. 
Bolívar, A., López, J. \& Murillo, F.J. (2013). Liderazgo en las instituciones educativas. Una revisión de líneas de investigación. Revista Fuentes, 14, pp.15-60.

Bolívar, A (2010). El Liderazgo Educativo y su Papel en la Mejora: Una Revisión Actual de sus Posibilidades y Limitaciones. Psicoperspectivas, 9(2), 9-33. doi:https://doi.org/10.5027/psicoperspectivas-Vol9-Issue2-fulltext-112

Bolívar, A. (2000). El liderazgo compartido según Peter Senge. En Liderazgo y organizaciones que aprenden (III Congreso Internacional sobre Dirección de Centros Educativos). Bilbao: ICE de la Universidad de Deusto, 459-471.

Bolívar, A. (2013). Políticas actuales de mejora y liderazgo educativo. Málaga: Aljibe.

Bracho, O., García, J. (2013). Algunas consideraciones teóricas sobre el liderazgo transformacional. Telos, 15 (2), pp. $165-177$

Cantón, I., \& Arias, A. R. (2008). La dirección y el liderazgo: aceptación, conflicto y calidad. Revista de educación, 345(1), 229-254

Carreño, I. (2008). Liderazgo distribuido: una nueva visión del liderazgo educativo. Universidad Autónoma. Madrid..

Castro, E., Miquilena, E., \& Peley, R. (2006). Las nuevas tendencias del liderazgo: hacia una nueva visión de las organizaciones educativas. Omnia, 12(1), 83-96.

Corbella, M. R. (2014). Liderazgo y responsabilidad educativa: el necesario liderazgo de directores y profesores en la educación. Revista Fuentes, (14), 85-104.

Davis, K. y Newstrom, J. (2003). Comportamiento humano en el trabajo (11. ${ }^{\text {a }}$ ed.). México: McGraw Hill Interamericana.

Day, C.; Sammons, P.; Leithwood, K; Hopkins, D.; Gu, Q.; Brown, E. y Ahtaridou, E. (2011). Succesful school leadership: Linking with learning and achievement. Londres: Open University Press.

Delgado, M. L. (2012). Las comunidades de liderazgo de centros educativos. Educar, 48(1), pp. 9-21.

Díez, E. J.; Valle, E.; Terrón, E. y Centeno, B. (2003). El liderazgo femenino y su ejercicio en las organizaciones educativas. Revista Iberoamericana de Educación, pp. 1-19

Fernández, J. M., \& Hernández, A. (2013). El liderazgo como criterio de calidad en la educación inclusiva. Estudios sobre educación, 24, 83-102.

Fernández, R. G., Palacios, S. G., \& Gutiérrez, V. J. O. (2016). Importancia de la dimensión formativa de los líderes pedagógicos. Revista Iberoamericana de Educação, 70, 131-144.

Franco, D. M. A., Ortega, C. G. L., \& Ortiz, A. M. G. (2016). El trabajo directivo en educación primaria: liderazgo, procesos participativos y democracia escolar. RECIE. Revista Electrónica Científica de Investigación Educativa, 3(1), 35-41.

Fundación Empresarios por la Educación. (2018). Apuntes para una discusión sobre liderazgo educativo. En línea: http://rle.fundacionexe.org.co/wp-content/uploads/2018/12/Liderazgo-educativo-271118.-Final.pdf

Furguerle Rangel, J., \& Vitorá, M. (2016). Liderazgo en los directivos de educación primaria. Telos, 18 (2), 208 $-227$.

García, I. M., \& Flores, J. G. (2018). Explicación de la satisfacción en la dirección escolar a partir del desempeño de la función directiva. Education in the Knowledge Society, 19(1), 77-95.

Gento, S. (2013). Relevancia del liderazgo de la dirección para la calidad de la institución educativa. Participación educativa, 2(2), 37-50.

Goleman, D. (2014). Liderazgo: el poder de la inteligencia emocional. Barcelona: B DE BOOKS.

Hallinger, P. (2005). Instructional leadership and the school principal: A passing fancy that refuses to fade away. Leadership and Policy in Schools, 4, 221-239. 
Hallinger, P. (2014). "Reviewing Reviews of Research in Educational Leadership: An Empirical Approach», Educational Administration Quarterly, 50(4), 539-576.

Hallinger, P. y Heck, R. H. (1998). Exploring the principal contribution to school effectiveness: 1980-1995. School Effectiveness and School Improvement, 9(2), 157-191.

Hallinger, P., \& Heck, R. H. (2014). Liderazgo colaborativo y mejora escolar: Comprendiendo el impacto sobre la capacidad de la escuela y el aprendizaje de los estudiantes. REICE. Revista Iberoamericana sobre Calidad, Eficacia y Cambio en Educación, 12(4), 71-88.

Hattie, J. (2015). Visible Learning: A Synthesis of Over 800 Meta-Analyses Relating to Achievement. London: Routledge.

Hernández, J. S., Tobón, S., \& Vázquez, J. M. (2015). Estudio del liderazgo socioformativo mediante la cartografía conceptual. RIEE. Revista Iberoamericana de Evaluación Educativa, 8(2), 105-128.

Hernández, M. E. M., Catrinao, J. C. C., \& Bravo, C. P. V. (2017). Creencias de directivos escolares: implicancias en el liderazgo pedagógico. Psicologia Escolar e Educacional, 21(3), 541-548.

Horn, A., \& Marfán, J. (2010). Relación entre liderazgo educativo y desempeño escolar: Revisión de la investigación en Chile. Psicoperspectivas, 9(2), 82-104.

Jiménez, I. V. (2017). Mirada de la comunidad educativa acerca del desempeño de directivas educativas: Algunas reflexiones. Revista Electrónica Educare, 21(1), 1-21

Leithwood, K. (2009) ¿Cómo liderar nuestras escuelas? Aportes desde la Investigación. Santiago de Chile: Fundación Chile.

Leithwood, K. \& Louis, K.S. (2011). Linking Leadership to Student Learning. San Francisco: Jossey-Bass.

Leithwood, K., Harris, A. \& Hopkins, D. (2008). Seven strong claims about successful school leadership. School Leadership \& Management, 28(1), 27-42.

Leithwood, K., Seashore, K., Anderson, S. \& Wahlstrom, K. (2004). How leadership Influences student learning. The Wallace Foundation. Recuperado de: http://www.wallacefoundation.org/knowledge-center/school-leadership/key-research/documents/how-leadership-influences-student-learning.pdf

Leithwood, K.; Mascall, B. \& Strauss, T. (2009). Distributed Leadership According to the Evidence. Londres: Routledge.

Llorent-Bedmar, V., Cobano-Delgado, V., \& Navarro-Granados, M. (2017). Liderazgo pedagógico y dirección escolar en contextos desfavorecidos. Revista Española de Pedagogía, 75(268), 541-564.

Lorenzo, M. (2005). El liderazgo en las organizaciones educativas. Revista Española de Pedagogía, 63 (232), 367-388.

Louis, K. S., Dretzke, B. \& Wahlstrom, K. (2011). How does leadership affect student achievement? Results from a national US survey. School Effectiveness and School Improvement: An International Journal of Research, Policy and Practice, 21(3), 315-336.

Maureira, Ó., Garay, S., \& López, P. (2016). Reconfigurando el sentido del liderazgo en organizaciones escolares contemporáneas: La perspectiva del liderazgo distribuido. Revista Complutense de Educación, 27 (2), pp. 689-706

Monarca, H. (2013). Fundamentos de las políticas educativas para el liderazgo organizacional. Análisis de un caso. Revista de Ciencias Sociales (Ve), 19(3), 523-535

Murillo, J., y Hernández, R. (2014). Liderando escuelas justas para la justicia social. Revista internacional de educación para la justicia social, 3(2), 11-30.

Navarro-Corona, C. (2016). Consideraciones teóricas sobre el concepto de liderazgo y su aplicación en la investigación educativa. Revista Educación, 53-66.

Navarro-Granados, M. (2017). Hacia un liderazgo educativo para la justicia social en las escuelas. Revista del Cisen Tramas/Maepova, 5 (1), 161-173. 
OCDE (2009). Creating Effective Teaching and Learning Environments: First Results from TALIS. Disponible en http://www.oecd.org/TALIS

OECD. (2008). Improving School Leadership. Paris: OECD

OECD. (2016). School Leadership for Learning: Insights from TALIS 2013. Paris: OECD Publishing

Ojeda, C. C. (2007). El liderazgo distribuido una apuesta de dirección escolar de calidad. REICE: Revista Iberoamericana sobre Calidad, Eficacia y Cambio en Educación, 5(5), 144-148.

Ord, K., Mane, J., Smorti, S., Carroll-lind, J., Robinson, L., Armstrong-Read, A., et-al (2013). Developing pedagogical leadership in early childhood education. Wellington: Te Tari Puna Ora o Aotearoa/NZ Childcare Association.

Pareja Fernández de la Reguera, J. (2009). Liderazgo y conflicto en las organizaciones educativas. Educación y Educadores, $12(1), 137-152$

Pedraja-Rejas, L., Rodríguez-Ponce, E., \& Villarroel González, C. (2012). Los estilos de liderazgo y la gestión de recursos humanos: una reflexión acerca del impacto sobre el desempeño de los estudiantes en la educación media. Ingeniare. Revista chilena de ingeniería, 20(3), 376-385

Pérez, V. V., Romero, E. P., Calderón, E. C., \& Verdú, F. M. M. (2012). Implementación de espirales de conocimiento y liderazgo situacional en la metodología b-learning. In X Jornades de Xarxes d'Investigació en Docència Universitària: la participació i el compromís de la comunitat universitària (pp. 2396-2412). Instituto de Ciencias de la Educación

Pont, B., Nusche, D., \& Moorman, H. (2008). Improving school leadership. Paris, OCDE. Disponible en: http://www. oecd.org/edu/schoolleadership

Ramos, C. P. F. (2015). El liderazgo de los equipos directivos y el impacto en resultados de los aprendizaje: un estudio de liderazgo educativo en las VI y VII regiones de Chile (Doctoral dissertation). Universidad de Granada

Redondo-Sama, G. (2015). Liderazgo dialógico en comunidades de aprendizaje. Intangible capital, 11(3), 437457.

Reviriego, M. (2013). Necesidades formativas de los directores escolares. In International Conference Re-conceptualizing the professional identity of the European teacher. Sharing Experiences (2013), p 434-450.

Risco, E. (2015). Interacción entre inteligencia emocional y estilos de liderazgo en directivos de instituciones educativas Tesis Doctoral. Universitat de València

Robinson, V. (2011). Student-centered leadership. San Francisco, CA: Jossey Bass.

Robinson, V. M. J. (2007). School leadership and student outcomes: identifying what works and why. Winmalee, NSW: Australian Council for Eduactional Leaders.

Robinson, V. M., Lloyd, C. A., \& Rowe, K. J. (2014). El impacto del liderazgo en los resultados de los estudiantes: Un análisis de los efectos diferenciales de los tipos de liderazgo. REICE. Revista Electrónica Iberoamericana sobre Calidad, Eficacia y Cambio en Educaciónm, 12(4e), 13-40

Rodas, H. D. C., \& Sipión, L. M. T. (2014). El liderazgo transformacional como apoyo en la gestión pedagógica del director. UCV-HACER. Revista de Investigación y Cultura, 3(2), pp.10-16

Rodríguez, A. Marmol, M. (2009). Liderazgo formativo y desarrollo sostenible de la calidad de la educación. Investigación \& Desarrollo, 17(1), 192-207.

Rodríguez, E. (2016). El liderazgo directivo en la hermenéutica micropolítica de la escuela. Alteridad, 11(1), 231 239.

Rodríguez-Molina, G. (2011). Funciones y rasgos del liderazgo pedagógico en los centros de enseñanza. Educación y Educadores, 14(2), 253-267

Salazar, M. (2006). El liderazgo transformacional ¿modelo para organizaciones educativas que aprenden? UNIrevista, 1(3), 1-12. 
Sánchez, M., \& Hernández-Castilla, R. (2014). Otros tiempos, nuevas visiones del liderazgo educativo: presentación. REICE: Revista Iberoamericana sobre Calidad, Eficacia y Cambio en Educación, 12(5), 5-8

Sánchez, R. U. (2014). Educación en acción: Un motor de cambio, liderazgo, compromiso social, innovación y creatividad. Calidad en la Educación Superior, 5(1), 18-33.

Sheppard, B.; Brown, J. \& Dibbon, D. (2009). School district leadership matters. Nueva York: Springer.

Sierra, G. M. (2016). Liderazgo educativo en el siglo XXI, desde la perspectiva del emprendimiento sostenible. Revista EAN, (81), 111-129

Theoharis, G. (2007). Social Justice Educational Leaders and Resistance: Toward a theory of social justice leadership. Educational Administration Quarterly, 43 (2), 221-258.

Thieme, C., \& Treviño, E. (2012). Liderazgo en educación: al final solo el carisma importa. Espacio abierto, 21(1), 37-55.

Tintoré, M. (2018). Líderes educativos y justicia social. Un estudio comparativo. Perspectiva Educacional, 57(2), 100-122.

Vallejo, E. G., Chancay, J. M. G., \& Mosquera, J. E. Q. (2016). Liderazgo y gerencia de proyectos educativos y sociales. Dominio de las Ciencias, 2(4), 274-283

Vázquez, E. B., \& Rodríguez, A. A. B. (2016). Programa de formación para el desarrollo del liderazgo educativo en los profesores de la facultad de Ciencias Económicas. Didasc@lia: Didáctica y Educación, 7(5), 97-110.

Vázquez, S., Bernal, J., \& Liesa, M. (2014). La conceptualización del liderazgo: una aproximación desde la práctica educativa. REICE. Revista Electrónica Iberoamericana sobre Calidad, Eficacia y Cambio en Educación. vol. 12, núm. 5, pp. 79-97

Villa, A. (2015). Importancia e impacto del liderazgo educativo. Padres y Maestros, 361, p. 6-11

Vivolo, C. (2015). Liderazgo creativo en los Directores Educativos del siglo XXI. Omnia, 21(1), 129-141. 